\title{
Drugs modulating the L-arginine:NO:cGMP pathway - current use in therapy
}

\author{
Magdalena Polakowska ${ }^{1}$, Jolanta Orzelska-Gorka ${ }^{2 *}$, Sylwia TalareK ${ }^{2}$
}

\begin{abstract}
${ }^{1}$ Student Research Group at the Chair and Department of Pharmacology and Pharmacodynamics, Medical University of Lublin, Chodzki 4A, 20-093 Lublin, Poland

${ }^{2}$ Chair and Department of Pharmacology and Pharmacodynamics, Medical University of Lublin, Chodzki 4A, 20-093 Lublin, Poland

\section{ARTICLE INFO}

Received 13 November 2015

Accepted 26 November 2015

\section{Keywords:}

nitric oxide,

vasodilators,

nebivolol,

sildenafil,

statins,

L-arginine.

ABSTRACT

Nitric oxide (NO) is a relatively novel messenger that plays a significant role in a wide range of physiological processes. Currently, it is known that, both, lack and excess of NO can cause diseases, thus a lot of substances have been discovered and utilized which can change the concentration of this molecule within the organism. The aim of the present work is to provide an overview of currently used agents modulating the L-arginine:NO:cGMP pathway, as well as to summarize current understanding of their pharmacological profiles. Nowadays, most of these agents are employed particularly in the treatment of cardiovascular diseases. Further studies can hold promise for enhancing the therapeutic equipment for a variety of other impairments, such as osteoporosis, and also in treatments of the central nervous system.
\end{abstract}

\section{INTRODUCTION}

Nitric oxide (nitrogen monoxide, NO) is a relatively novel messenger that plays a significant role in a wide range of physiological processes. In 1978, Feride Murad showed that nitroglycerine is a substrate for $\mathrm{NO}$ and increases the level of cyclic guanosine-3', $5^{\text {' }}$-monophosphate (cGMP) in tissues. In 1980, Robert F. Furchgott indicated that the endothelium released an agent that brought about the relaxation of the central smooth muscles. This he called "endotheliumderived relaxing factor" (EDRF). Louis J. Ignarro found later that EDRF was, in fact, $\mathrm{NO}$ [6]. Their discoveries have shown the importance of $\mathrm{NO}$ as a signaling molecule in the cardiovascular system. In 1998, the Nobel Prize in Physiology or Medicine was awarded to these three scientists "for their discoveries concerning the nitric oxide as a signaling molecule in the cardiovascular system" [48]. This was the beginning of precious studies on the role of $\mathrm{NO}$ as a biological mediator. Currently, it is known that, both, lack and excess of NO can induce a variety of diseases, thus, work has been undertaken in discovering a lot of substances which bring about changes in the concentration of this molecule within the organism.

$\mathrm{NO}$ is an important gaseous chemical messenger which is extremely labile, and has a very short half-life. For this

\footnotetext{
* Corresponding author

e-mail: jolanta.orzelska@umlub.pl

tel. 814487256
}

reason, it is quickly converted into nitrates and nitrites by oxygen and water [24]. It is also a free radical that through a binding of the 8-electron atom of oxygen with the 7-electron atom of nitrogen, has 1 unpaired electron [6]. This molecule plays a meaningful role in various physiological processes in the body. These are mainly associated with the cardiovascular, immune and nervous systems, but also can be engaged in certain pathological responses, e.g. in hypertension, established ischemic heart disease (lack of NO) and septic shock or inflammation (NO in excess) [35].

NO is generated by a family of NO synthases (NOS) that convert L-arginine as the substrate, to NO and L - citrulline, in the presence of molecular oxygen and reduced nicotinamide-adenine-dinucleotide phosphate as co-substrates. Flavin adenine dinucleotide, flavin mononucleotide, and (6R-)5,6,7,8-tetrahydrobiopterin are cofactors of all NOS/isoenzymes [15, Fig.1]. NO is synthesized by three genetically different synthases: endothelial NOS (eNOS), neuronal NOS (nNOS) (which are constitutively expressed) and inducible NOS (iNOS). These are synthesized following induction by pro-inflammatory cytokines or endotoxin [51]. The presence of nNOS was found in blood platelets, neurons, synaptic spines, cardiac, skeletal and smooth muscles, while eNOS is mainly located in the endothelial cells. Those constitutive isoforms are dependent on calmoduline and factors increasing $\mathrm{Ca}^{2+}$ concentration. In contrast, iNOS is mainly found in the microglia, astrocytes, 
macrophages and neutrofiles. It is not $\mathrm{Ca}^{2+}-$ calmoduline - dependent, but its long activity defines the concentration of cytokines, as well as inflammatory and immunological stimulation $[16,17]$.

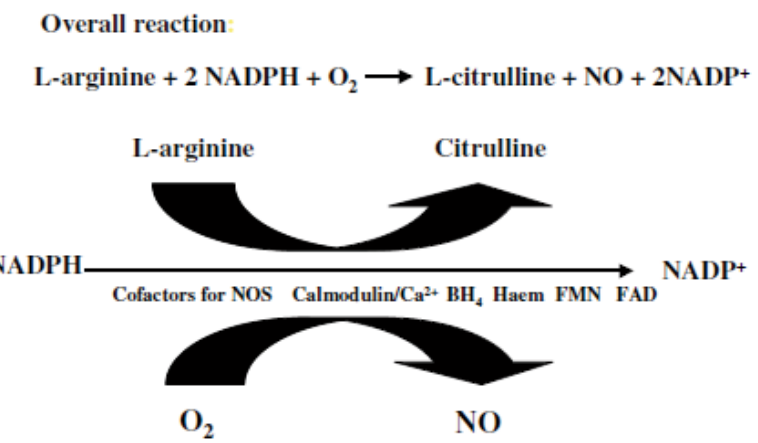

Figure 1. Biosynthesis of NO (nicotinamide-adenine-dinucleotide phosphate (NADPH); flavin adenine dinucleotide (FAD), flavin mononucleotide (FMN), and (6R-)5,6,7,8-tetrahydrobiopterin (BH4) [4]

NO functions as a neurotransmitter in the peripheral and central nervous system (CNS), however, it is not like the typical neurotransmitter stored in the synaptic vesicles, but after its synthesis, it is quickly released to the synaptic cleft. NO diffuses out between neurons, whereas a typical neurotransmitter is released in the process of exocytosis [40]. In addition, it does not interact with the receptors on the neural membranes, but directly binds to soluble guanyl cyclase (sGC) - one of the most specific targets for NO [7]. NO interacts with the haem iron at the active site of sGC, and, during this process, induces a modification of conformation in the haem structure. The result of this binding is the increase in quantity of cGMP. This, in turn, activates cGMP-dependent protein kinases, ion channels and phosphodiesterases (PDE), and, subsequently, gets up to protein phosphorylation. The cGMP compound plays a central role in NO signaling and in the regulation of physiologic responses. Furthermore, the L-arginine:NO:cGMP signaling pathway takes part in blood pressure regulation, synaptic plasticity, atypical neurotransmission, penile erection in the presence of nNOS; non-specific immune defense, mediation of inflammation and septic shock in the presence of iNOS; as well as vasodilation, vasoprotection and prevention of atherosclerosis in the presence of eNOS $[7,15]$.

The aim of the present work is to provide an overview of currently used agents modulating L-arginine:NO:cGMP pathway, and to summarize current understanding of their pharmacological profiles.

\section{NO IN CARDIOVASCULAR DISEASES (CVD)}

$\mathrm{NO}$ is a substance which is responsible for the vascular relaxation induced by acetylcholine and other endotheliumdependent vasodilators, doing so via stimulation of the sGC and then growth of cGMP concentration. NO in the cardiovascular system, is mainly formed by eNOS, and it functions as a regulator of vasomotor tone by lowering the intracellular calcium content. This leads to relaxation of the vascular smooth muscle. It also inhibits platelet aggregation and adhesion. Moreover, it suppresses degranulation of mastocytes [35]. In high concentrations, NO inhibits the proliferation of smooth muscle cells [36]. Furthermore, it is involved in the reaction between the body's white blood cells and the membranes of blood vessel walls. These findings established NO as a homeostatic regulator in the cardiovascular system. In addition, NO plays a role in a number of physiological conditions and pathological states with regard to the system. Among these are atherosclerosis, hypertension and vasoconstriction [35]. Therefore, endothelial dysfunction results in NO deficiency and the uncoupling of NOS generating free radicals [24].

CVD are a group of disorders that include congestive heart failure (CHF) and ischemic heart disease. Such disorders can subsequently lead to angina pectoris or even acute myocardial infarction, and are characterized by vasoconstriction. Ischemic myocardium appears when the supply of oxygenated blood through the coronary arteries becomes inadequate to meet the demands of the cardiac muscle, leading to myocardial hypoxia [39]. Acute and chronic ischaemia results in the destruction of the endothelium. This may restrict vasodilation or promote vasoconstriction. The cause of CVD is found to be a shortage of NO, and this situation can confine the NO-dependent signal pathways [9,39]. In CVD therapy, substances with vasorelaxant properties, as well as other cardiovascular agents modulating endogenous NO bioactivity, are used [24]. Such nitrovasodilators consist of metabolic donors (organic nitrates and nitrites, nicorandil) and direct donors (sodium nitroprusside (SNP), NO gas and molsidomine) [49]. Moreover, NO may also interfere with $\beta$-blockers and with the renin-angiotensinaldosterone system (RAAS) [34,41].

\section{Nitrates and nitrites}

Organic nitrates and nitrites have similar properties, but they have different mechanisms of action and different enzymatic requirements for NO generation. Glyceryl trinitrate (nitroglycerine), isosorbide dinitrate, and isosorbide5-mononitrate - the most commonly used organic nitrates - are effective in reducing ventricular preload, by increasing peripheral venous capacity [39]. In the vascular smooth muscle cells, they are metabolized to 1,2-glyceryl dinitrate and nitrite, via mitochondrial aldehyde dehydrogenase-2, and then to NO and S-nitrosothiols [26]. Current indications for the need to undergo nitrate therapy include chronic stable angina pectoris, unstable angina pectoris, acute coronary syndrome, stable coronary artery disease and acute or chronic CHF [37]. Nitroglycerine is a very popular drug used in clinical practice, particularly for the treatment of angina, because of its quick vasodilation properties and its abating of the heart's overload [5]. These effects are accomplished by its reduction of the peripheral return of blood to the heart, and by its lessening of the resistance to the outflow of blood from the heart into the main arterial circulation. It also moderates cardiac preload, decreases myocardial wall tension and oxygen requirements, as well as increases myocardial oxygen supply by way of its vasodilatory effects [20]. Nitroglycerine provides rapid therapeutic relief of acute recurrent angina attacks. It can also be used prophylactically when angina is anticipated, so as to increase angina-free 
walking time on treadmill testing and to increase exercise tolerance [5].

Amyl nitrite - the most popular nitrite - is also a source of NO. At low $\mathrm{pH}$, nitrites are a source of nitrous acid that can give rise to the spontaneous generation of $\mathrm{NO}$ : $2 \mathrm{HNO}_{2} \rightarrow \mathrm{H}_{2} \mathrm{O}+\mathrm{N}_{2} \mathrm{O}_{3}$ and $\mathrm{N}_{2} \mathrm{O}_{3} \rightarrow \mathrm{NO}+\mathrm{NO}_{2}$ [8]. Amyl nitrite, as an antihypertensive medicine, may be employed in the treatment of heart diseases such as angina, as well as in the treatment of cyanide poisoning. Recently, it was indicated that the nitrite anion has additional uses. Among these are modulating hypoxic vasodilation and providing cytoprotection in ischemia-reperfusion injury. Other organic nitrites (isoamyl nitrite and isobutyl nitrite) also demand enzymatic catalysis by vascular cellular subfractions in order to generate NO. Currently, nitrites are not used in the treatment of CVD - hypertension or ischemic heart disease [8,39].

Nitrovasodilators act through conversion to NO. This results in vasodilation, whereas chronic therapy may lead to pharmacological tolerance. While all of the organic nitrates have potent short-term vascular, hemodynamic, and clinical effects [42], current evidence indicates that long acting formulations, high doses, or frequent dosing regimens are particularly likely to induce vascular tolerance to nitrates which then limits their clinical action [37].

Nitrates increase vascular superoxide production and rapidly format peroxynitrite. This, in turn, can enhance oxidative stress and endothelial dysfunction. Moreover, this impairs NO bioavailability and also reduces the responsiveness of its action within smooth muscle cells [42]. The tolerance to nitrates can, in addition, bring about a depletion of the sufhydryl groups during the conversion of nitroglycerine to NO, as well as defective cGMP activity - due to desensitization of cGC, enhanced PDE activity and also defective regulatory system activity [49]. Of note, while the reduction of endothelial NO production following the administration of organic nitrates (such as nitroglycerine), may be a mechanism of nitrate tolerance, amyl nitrite has different vascular actions and hemodynamic effects, hence, it induces less tolerance, in comparison with nitroglycerine [39].

\section{Nicorandil}

Nicorandil is an adenosine triphosphate sensitive potassium $\left(\mathrm{K}_{\text {ATP }}\right)$ channel opener, and, thus, it has vasodilator properties. Its vasodilating properties are attributable to a nitrate group within its chemical structure - it is a NO donor. Both mechanisms of its action contribute to vasodilation, but are independent. At lower concentrations, nicorandil activates the $\mathrm{K}_{\text {ATP }}$ channel, while at higher concentrations, it acts through NO donation $[21,22]$. Nicorandil brings about sustained vasodilation, it markedly relaxes coronary vascular smooth muscle and increases the coronary blood flow. Therefore, it is used in the treatment of various types of angina pectoris and ischemic symptoms. In this, its general efficacy is similar to nitrate administration. Currently, nicorandil is not approved in the USA, it is only available in several European and Asian countries. In a chemical aspect, this molecule belongs to the organic nitrate groups. This categorization is done on the basis of the fact that it has a nitrate moiety [21]. However, at clinical doses, nicorandil, unlike nitroglycerine or isosorbide dinitrate, possesses a weaker hemodynamic effect on blood pressure, heart rate or cardiac contractility, yet it still yields anti-anginal effects [43]. In the ischemic myocardium, nicorandil has revealed cardioprotective effects. This is demonstrated by reduced myocardial necrosis and improved myocardial stunning [22] following administration. Angina pectoris is brought about by transient episodes of myocardial ischemia which create an imbalance in the myocardial oxygen supply and demand relationship. Hence, Nicorandil is commonly used to treat this disease because of its dual mechanism of action. Moreover, its action differs significantly from currently available vasodilators by its inducing a sustained dilation of both the venous capacitance and arterial resistant vessels, thus reducing preload and afterload [21].

\section{Direct donors}

The NO direct donors - SNP, NO gas and molsidomine - release NO spontaneously, as defined via the range of redox forms of nitrogen monoxides generated by the donor molecule [24].

SNP is a water-soluble sodium salt comprised of $\mathrm{Fe}^{2+}$ complexed with NO and five cyanide ions, thus, during spontaneous decomposition, it releases anions and NO. Additionally, this molecule may react with the sulfhydryl groups found within the erythrocytes, albumin, and other proteins, so as to release NO. SNP is a very potent nitrovasodilator, and is used for the treatment of hypertensive crises and heart failure [23]. Hypertension is characterized by a constriction of the coronary arteries that leads to a sustained increase in systemic arteriolar tone, when compared with normotensive subjects. Herein, the reduction in release of NO is found to be the cause of hypertension. Thus, SNP, in donating NO, leads to rapid arterial, venous dilatation and the acute lowering of blood pressure [9]. However, SNP can be broken down by hemoglobin into cyanide. Acute cyanide toxicity is observed when cyanide ions bind to tissue cytochrome oxidase, and, thus, limit oxygen utilization in essential tissues. Cyanide toxicity manifests as lactic acidosis, progressing to cardiovascular collapse and to an increase of venous oxygen. Furthermore, thiocyanate may be generated during the detoxification of cyanide. This, itself, causes toxicity. These anions may also be transported to the liver where they react with thiosulfate and cobalamin to form thiocyanate $[23,47]$. Herein, the thiocyanate toxicity can bring about nausea, fatigue and CNS depression [47].

Other molecules which directly increase the concentration of NO within the body are those of gaseous $\mathrm{NO}$, which is applied via inhalation (inhaled $\mathrm{NO}-\mathrm{iNO}$ ). This iNO is used for the treatment of adult respiratory distress syndrome, and in term and near-term ( $>34$ weeks) neonates with hypoxemic respiratory failure [11]. Adult respiratory distress syndrome is characterized by indications of intrapulmonary shunting that results in arterial hypoxemia, and by acute pulmonary arterial hypertension due to vasoconstriction and widespread occlusion of the pulmonary microvasculature [44]. Hypoxemic respiratory failure is then manifested by increases in the ratio of pulmonary vascular resistance resulting from vasoconstriction, structural remodeling of the pulmonary vasculature and intravascular obstruction, which then lead to hypoxemia [31]. Administering iNO can reduce pulmonary 
hypertension and improve arterial oxygenation. It selectively dilates blood vessels in only those lung segments that are actively participating in gas exchange at the alveolar-capillary level, thus it increases blood flow in these areas, and results in improvement of ventilation to perfusion matching. In addition, iNO administration reduces the use of extracorporeal membrane oxygenation, and incidences of death [1]. The product also has a short half-life that minimizes systemic effects. In addition, such iNO therapy is well tolerated, safe, and relatively easy to administer [25].

Molsidomine is a pro-drug for the formation of $\mathrm{NO}$, and is classified as being a sydnone. It is also a direct NO donor, particularly when used in the treatment of stable angina pectoris, acute myocardial infarction, pulmonary hypertension and CHF [20]. It acts via its metabolite - 3-morpholinosydnonimine $(\mathrm{SIN}-1)$. This has a pharmacologically active nitrate group and releases NO [43]. SIN - 1 acts as a potent hypotensive and spasmolytic agent that is without influence on the autonomic nervous system. It only interacts directly with vascular smooth muscles and has a negative inotropic effect, reducing cardiac preload and afterload, as well as peripheral blood pressure [46].

\section{Nebivolol}

Nebivolol is a third-generation highly selective blocker of $\beta_{1}$-adrenergic receptors that demonstrates nonadrenergic vasodilating properties. It can stimulate the endogenous production of NO by inducing phosphorylation of the eNOS. Indeed, it is the only drug in the group of $\beta$-blockers with this ability. Nebivolol is a 1:1 racemic mixture of $\mathrm{D}$ - and L-enantiomers, of which D-nebivolol is considered to be a highly selective $\beta_{1}$-adrenergic receptor antagonist, whereas L-isomer determines the stimulation of eNOS and subsequent endothelium-dependent vasodilation [36]. Besides the vasorelaxant properties resulting from this dual action, it is also able to reverse endothelial dysfunction. Nebivolol is employed in CVD therapy, mainly for hypertension. This is because this drug has an agonist action on the $\beta 3$ receptors mediating the production of $\mathrm{NO}$ in the heart, hence, resulting in protection against $\mathrm{CHF}$ [34].

Nebivolol is used for the treatment of hypertension in the USA, and for hypertension and heart failure in Europe [14]. The Nebivolol effect occurs after 2 to 8 weeks of hypertension therapy [36]. It can be administered both as monotherapy and in combination with other classes of antihypertensive agents. It is considered to be a safe and welltolerated drug that possesses a tolerability profile similar to other of $\beta$-blockers. Of note, its antihypertensive effect depends on the reduction of myocardial contractility, heart rate, and cardiac output $[12,14]$. In the protection against heart failure, nebivolol, by lowering heart rate, reduces the myocardial workload, decreases the potential of sudden death through suppression of arrhythmias and improves cardiac performance [14]. Nebivolol has similar efficacy in vasorelaxation as does inhibitors of the RAAS, as well as does calcium channel blockers. It also has comparable properties to other $\beta$-blockers, but demonstrates fewer side effects than do older generations of $\beta$-blockers. It should be noted that this agent does not increase the risk of diabetes mellitus, atherogenic dyslipidemia or weight gain [12].

\section{NO AND RAAS}

The RAAS is an important hormonal system that has impact upon the cardiovascular, renal and adrenal gland functions, by way of regulating the volume of extracellular fluid, the sodium and potassium balance and the blood pressure. The abnormal activity of the RAAS leads to the enhancement of oxidative stress, and particularly to superoxide anion $\left(\mathrm{O}_{2}^{-}\right)$formation. This can increase vasoconstriction and sodium reabsorption in kidneys. In turn, it may result in the development of hypertension, CHF, stroke or renal disease. The RAAS has also pro-inflammatory and profibrotic effects at the cellular and molecular levels [29, 41]. What is more, the activation of the RAAS results in the subsequent activation of angiotensin II which markedly can induce both $\mathrm{O}_{2}{ }_{2}^{-}$and $\mathrm{NO}$ formulation. Angiotensin II stimulates the expression of NOS and NO increase via the angiotensin II type II (AT2) receptor that is responsible for a vascular protective mechanism. However, NO down-regulates the angiotensin II type I (AT1) receptor effects, and this may be one of the mechanisms of the antiatherogenic and antihypertensive properties that NO exhibits [10]. In addition, NO regulates vascular homeostasis and inhibits the renal tubular transport of sodium, and, subsequently, plays an important role in the excretory function of the kidneys [29]. Furthermore, Angiotensin II brings about constriction and enlargement of the vascular smooth muscle, while these muscles can be dilated in response to NO. Angiotensin II and NO can also antagonize each other in many vascular functions, such as in cell growth, apoptosis, and inflammation [10]. Of note, the imbalance of interaction in the RAAS, between $\mathrm{NO}$ and $\mathrm{O}_{2}^{-}$, induces the development of several vascular diseases, as the RAAS may increase the level of reactive oxygen species present and may induce an oxidative stress that can inactivate NO. Furthermore, endothelial dysfunction can also reduce NO concentration [29]. Therapeutic strategies for CVD would include agents that enhance NO activity, and agents that reduce the abnormal activity of the RAAS.

\section{NO IN ERECTILE DYSFUNCTION AND PULMONARY HYPERTENSION}

Sildenafil, tadalafil, vardenafil and avanafil are PDE type 5 (PDE 5) inhibitors that are used as a first-line therapy for the treatment of erectile dysfunction. This disease is defined by the National Institute of Health as the incapacity to achieve and sustain a satisfactory erection for the purposes of sexual intercourse [18]. The inhibition of PDE 5 results in a blockade of cGMP hydrolysis that leads to an increase of cGMP level, and, subsequently, to a growth in NO concentration. NO is responsible for vasodilation in the corpus cavernosum - a dilation of the smooth muscle and blood vessels in the penis that brings about a stiff erection. This group of drugs is characterized by having a tolerable sideeffect profile, by effectiveness and quick onset of action $[13,18]$. Of interest, their administration could enhance NOmediated vasodilation in other vascular beds and improve endothelial function [18]. 
Although all current PDE 5 inhibitors work by the same mechanism, they work at different plasma concentrations and have different selectivity with regard to the PDE 5 isozyme. Sildenafil is a reversible PDE 5 inhibitor and it has been employed in therapy since 1998. Besides erectile dysfunction therapy, it can selectively utilized in reducing pulmonary vascular resistance, as well as in improving pulmonary artery pressure and physical exercise tolerance. These effects are of importance in the treatment of persistent pulmonary hypertension, as characterized by progressive increase in the pulmonary pressure $[4,18]$. In the therapy of persistent pulmonary hypertension of the newborns, iNO is commonly applied. However, administration of sildenafil has shown to be effective, and is associated with an enhancement of the oxygenation and a reduction in mortality with no clinically significant side effects. Thus, it can be used either as a supplement to iNO therapy, or as an alternative to iNO [50]. Sildenafil can also generate platelet anti-aggregatory activity and inhibit thrombus formation. Furthermore, its administration improves eNOS activation. This results in a reduction of the oxidative stress brought about by hyperglycemia and by insulin resistance conditions [29].

\section{NO AND STATINS}

The bioavailability of the NO is also regulated by statins. They promote eNOS activity and prevent free-radicals inducing NO inactivation. At therapeutic doses, statins have pleiotropic anti-aggregative, anti-inflammatory and anti-oxidant properties. In addition, their administration can improve vasodilation and promote migration and survival of endothelial cells by stimulating protein kinase $\mathrm{B}$ and $\mathrm{NO}$ formulation [33]. Furthermore, statin therapy can lower the cholesterol concentration by inhibiting 3-hydroxy-3methylglutaryl-CoA (HMG-CoA) reductase (an enzyme that controls cholesterol biosynthesis), and by up-regulating hepatic low density lipoprotein (LDL) receptors $[33,45]$. Of note, as atherosclerosis is initiated by a reduction in NO concentration due to impaired endothelial function, and LDL-cholesterol decreases eNOS activity, statins may reverse this effect as the result of their dual action mechanism. They also may be used in the prevention and treatment of other CVD's, including hypertension and coronary heart disease [45].

\section{L-ARGININE SUPPLEMENTATION}

L-arginine is naturally produced and stored in significant amounts within intracellular depots in the body, however, oral supplementation or infusion of this molecule increases substrate availability. This subsequently promotes eNOS expression and activity. In consequence, as a metabolic precursor of NO, L-arginine enhances peripheral endotheliumdependent dilatation, and it inhibits platelet aggregation. Hence, it is administered to reduce the symptoms resulting from a decrease of NO concentrations in patients with $\mathrm{CHF}$ $[19,32]$. In addition, short-term intracoronary administration of L-arginine improves coronary endothelial response to acetylcholine, in hypercholesterolemia [32].

\section{NO IN OSTEOPOROSIS}

It is known that relative NO deficiency, secondary to estrogen shortage, in postmenopausal women, and perhaps in older men, is responsible for osteoporosis. These findings have guided research efforts towards NO therapy as treatment for post-menopausal osteoporosis [46]. The clinical trials that have been performed so far, however, have returned varied results. In a one-year clinical trial carried out by Wimalawansa et al., nitroglycerin was administered topically. The authors observed that nitroglycerin was as effective as estrogen replacement therapy in preventing bone loss. A second three-year study examined the effect of nitroglycerin administered daily to post-menopausal women. Herein, no difference was observed between the control and the nitroglycerin groups [38]. Most recently, Jamal et al. conducted a two-year clinical trial that demonstrated that topical nitroglycerin therapy on post-menopausal subjects reduced bone resorption and increased bone mineral density. The authors concluded that a systemic NO administration regimen may be an effective treatment option for osteoporosis [38].

\section{NO IN SEPTIC SHOCK}

Excessive release of NO plays a significant role in the pathogenesis of septic shock. Such shock can be toxic because it inactivates the mitochondrial respiratory chain enzymes and can induce apoptosis in the target cells [17]. A low level of NO is constitutively (by way of eNOS and nNOS) produced in vascular endothelial cells, and NO regulates the relaxation of smooth-muscle, and, hence, blood pressure. However, under inflammatory or septic conditions, an iNOS is expressed. This generates an uncontrolled NO production that is responsible for hypotension, for vascular hypo-reactivity to vasoconstrictors, organ injury, and the dysfunction of the defense system [30].

The inhibition of either the expression of the iNOS protein (e.g., with dexamethasone) or of NOS activity (e.g., with inhibitors of iNOS activity) exerts beneficial effects in septic shock. The effect of administering nonselective NOS inhibitors, such as $\mathrm{N}^{\mathrm{G}}$-L-arginine-monomethyl and $\mathrm{N}^{\mathrm{G}}$-methyl-L-arginine hydrochloride, has been studied in patients with acute circulatory failure due to severe sepsis. In the therapy of septic shock, nonselective NOS inhibitors have been seen to inhibit iNOS, and, subsequently, to block the massive production of NO, hence, to reverse its negative actions [35]. Additionally, they increase basal coronary vascular resistance and blunt the vasodilator response to the vasodilator agonists acetylcholine and bradykinin, which, in normal conditions, engage specific endothelial cell receptors that stimulate the production of NO and other vasodilator substances $[6,9]$.

\section{NO AND NONSTEROIDAL ANTI-INFLAMMATORY DRUGS (NSAIDS)}

NSAIDs have been seen to be useful in the management of pain and inflammation, and are widely employed for the treatment of a number of inflammatory conditions, among 
these, rheumatoid arthritis and osteoarthritis. However, they also elicit severe gastrointestinal and cardiovascular side effects. NSAIDs engender their clinically beneficial effects by acting as non-selective inhibitors of both cyclooxygenase $(\mathrm{COX}) 1$ and COX2 isoenzymes, therefore, their administration blocks the production of prostanoids and thromboxanes. The ability to inhibit prostanoid production has also been suggested to be the cause of their severe gastrointestinal side effect. The awareness of the protective effects of NO on the gastrointestinal mucosa has led to the development of a new class of compounds, the cyclooxygenase-inhibiting NO Donors (CINOD). These compounds are structurally based on known NSAIDs, but are chemically linked to a NO donating-moiety [2]. Naproxcinod was the first of a novel class of drugs referred to as the CINOD. Naproxcinod is a prodrug that becomes metabolized to the nonselective COX NSAIDs, naproxen and butanediol mono-nitrate, and metabolized to NO, and, subsequently, to the vasodilator nitrate. The rationale for this approach was to help restore the protective effects of prostaglandins via NO, which stimulates many protective factors adversely affected by COX inhibition - including bicarbonate secretion, mucus production and gastric mucosal blood flow. Three phase III trials in patients with osteoarthritis compared the administration of naproxcinod, naproxen, and placebo over 13 weeks, and demonstrated naproxcinod superiority and efficacy over placebo. However, the Food and Drug Administration (FDA) denied new drug application (NDA) for naproxcinod, in 2010 , as they concluded that a longer trial was necessary to further evaluate its effect on blood pressure and gastrointestinal tract safety [3].

\section{CONCLUSION}

NO is a significant biological mediator that is needed for regulating the functions of the cardiovascular and immune systems, as well as the CNS. NO concentration is dependent on the modulation of the L-arginine:cGMP:NO pathway. A lack or an excess of this molecule may engender many types of disorders, among these, CVD, septic shock, osteoporosis, erectile dysfunction and persistent pulmonary hypertension. Therefore, nowadays, a wide variety of approved NO bioavailability modulators are utilized in medicine, most of which are employed particularly in treating CVD.

Additionally, it should be noted that the L-arginine: NO:cGMP pathway was found to play an important role in brain functioning. Thus, modulators of this pathway could be considered as being potential agents of therapy of CNS disorders such as anxiety and aggression. It is known that NO can promote neurogenesis, reduce neurological deficits after stroke, and improve memory retention and attention, as well. Based on these features, NO modulators can be proposed as being potentially useful agents in treating Alzheimer's disease, seizures and pain. Nevertheless, the current findings regarding $\mathrm{NO}$ and its role in the CNS are still contradictory, hence, further research is needed.

A summary of the effects of NO agents that are employed in present day therapy is provided in Table 1.

Table 1. Current use of drugs modulating the L-arginine:NO:cGMP pathway

\begin{tabular}{|c|c|c|c|c|}
\hline Group & Examples & Action & Result & Use in treatment \\
\hline Nitrates & $\begin{array}{c}\text { Nitroglycerine, isosorbide } \\
\text { dinitrate, isosorbide-5- } \\
\text { mononitrate }\end{array}$ & $\begin{array}{c}\text { Metabolism to } 1,2 \text {-glyceryl dinitrate } \\
\text { and nitrite, via mitochondrial } \\
\text { aldehyde dehydrogenase-2 and } \\
\text { then to NO }\end{array}$ & Increase in NO concentration & $\begin{array}{c}\text { Stable and unstable angina } \\
\text { pectoris, acute coronary syndrome, } \\
\text { stable coronary artery disease and } \\
\text { acute and chronic CHF }\end{array}$ \\
\hline $\begin{array}{l}\text { Nitrate, KATP } \\
\text { channel activator }\end{array}$ & Nicorandil & $\begin{array}{l}\text { Metabolism to NO like other } \\
\text { nitrates; opening of the KATP } \\
\text { channel }\end{array}$ & $\begin{array}{l}\text { Increase in NO concentration; } \\
\text { hyperpolarization of membranes }\end{array}$ & $\begin{array}{c}\text { Angina pectoris and ischemic } \\
\text { symptoms (in several European and } \\
\text { Asian countries) }\end{array}$ \\
\hline Direct donors & $\begin{array}{l}\text { iNO, SNP, molsidomine } \\
\quad(\text { and SIN - 1) }\end{array}$ & Spontaneous release of NO & Increase in $\mathrm{NO}$ concentration & $\begin{array}{l}\text { iNO: adult respiratory distress } \\
\text { syndrome, in term and near- } \\
\text { term neonates with hypoxemic } \\
\text { respiratory failure; SNP: } \\
\text { Hypertension, heart failure; } \\
\text { molsidomine: angina pectoris, } \\
\text { acute myocardial infarction, } \\
\text { pulmonary hypertension, CHF }\end{array}$ \\
\hline$\beta$-blocker & Nebivolol & $\begin{array}{c}\text { Induction of eNOS activity, selective } \\
\beta 1 \text { - receptors antagonist; } \beta 3 \\
\text { receptors agonist }\end{array}$ & $\begin{array}{c}\text { Increase in NO concentration; } \\
\text { suppression of the renin-angiotensin } \\
\text { system; inhibition of the sympathetic } \\
\text { system }\end{array}$ & $\begin{array}{l}\text { Hypertension (in the USA), } \\
\text { hypertension and heart failure (in } \\
\text { Europe) }\end{array}$ \\
\hline PDE 5 inhibitors & $\begin{array}{l}\text { Sildenafil, tadalafil, } \\
\text { vardenafil, avanafil }\end{array}$ & $\begin{array}{l}\text { Blockade of cGMP hydrolysis, } \\
\text { increase in cGMP level }\end{array}$ & Increase in NO concentration & $\begin{array}{l}\text { erectile dysfunction, persistent } \\
\text { pulmonary hypertension }\end{array}$ \\
\hline Statins & $\begin{array}{l}\text { Atorvastatin, fluvastatin, } \\
\text { lovastatin, simvastatin }\end{array}$ & $\begin{array}{l}\text { Induction of eNOS activity, } \\
\text { prevention of NO inactivation by } \\
\text { radicals, inhibition of HMG-CoA } \\
\text { reductase, decrease in density of } \\
\text { LDL receptors }\end{array}$ & $\begin{array}{c}\text { Increase in NO concentration, } \\
\text { stimulation of protein kinase } \\
\mathrm{B} \text {, decrease in the cholesterol } \\
\text { concentration }\end{array}$ & $\begin{array}{l}\text { Prevention and treatment of CVD, } \\
\text { mainly hypertension, coronary } \\
\text { heart disease, atherosclerosis }\end{array}$ \\
\hline Substrate for NO & L-arginine & Induction of eNOS activity & Increase in NO concentration & CVD like CHF, hypercholesterolemia \\
\hline $\begin{array}{l}\text { Inhibitor of the } \\
\text { NO synthesis }\end{array}$ & $\begin{array}{c}\text { NG-L-arginine-monomethyl } \\
\text { and NG-methyl-L-arginine } \\
\text { hydrochloride }\end{array}$ & Inhibition of iNOS & Decrease in NO concentration & Septic shock \\
\hline
\end{tabular}

\section{REFERENCES}

1. Akmal A.H., Hasan M.: Role of nitric oxide in management of acute respiratory distress syndrome. Ann. Thorac. Med., 3, 100-103, 2008.

2. Amoruso A. et al.: Characterization of the anti-inflammatory properties of NCX 429, a dual-acting compound releasing nitric oxide and naproxen. Life Sci. 126, 28-36, 2015.
3. Atkinson T.J. et al. What's new in NSAID pharmacotherapy: oral agents to injectables. Pain Med. 1,S11-7, 2013.

4. Barnett C.F., Machado R.F.: Sildenafil in the treatment of pulmonary hypertension. Vasc. Health Risk. Manag., 2, 411-422, 2006. 
5. Boden W.E. et al.: Role of short-acting nitroglycerin in the management of ischemic heart disease. Drug. Des. Devel. Ther., 9, 4793-4805, 2015.

6. Bruckdorfer R.: The basics about nitric oxide. Mol. Aspects Med., 26, 3-31, 2005.

7. Buchwalow I. et al.: L-arginine-NO-cGMP signalling pathway in pancreatitis. Sci. Rep., 3, 1899, 2013.

8. Butler A.R., Feelish M.: Therapeutic uses of inorganic nitrite and nitrate: from the past to the future. Circulation, 117, 2151-2159, 2008.

9. Cannon R.O.: Role of nitric oxide in cardiovascular disease: focus on the endothelium. Clin. Chem., 44, 1809-1819, 1998.

10. Chen Y. et al.: Functional interplay between angiotensin II and nitric oxide: cyclic GMP as a key mediator. Arterioscl. Throm. Vas., 23, 26-36, 2003.

11. DiBlasi R.M., Myers T.R., Hess D.R.: Evidence-based clinical practice guideline: inhaled nitric oxide for neonates with acute hypoxic respiratory failure. Respir. Care., 55, 1717-1745, 2010.

12. DiNicolantonio J.J. et al.: $\beta$-Blockers in hypertension, diabetes, heart failure and acute myocardial infarction: a review of the literature. Open Heart, 2, 2015.

13. Evans J.D., Hill S.R: A comparison of the available phosphodiesterase- 5 inhibitors in the treatment of erectile dysfunction: a focus on avanafil. Patient. Prefer. Adherence, 9, 1159-1164, 2015.

14. Fongemie J., Felix-Getzik E.: A Review of Nebivolol Pharmacology and Clinical Evidence. Drugs, 75, 1349-1371, 2015.

15. Förstermann U., Sessa W.C.: Nitric oxide synthases: regulation and function. Eur. Heart J., 33, 829-837, 2012.

16. Garry P. S. et al.: The role of the nitric oxide pathway in brain injury and its treatment - From bench to bedside. Exp. Neurol., 263, 235-243, 2015.

17. Guix F. X. et al.: The physiology and pathophysiology of nitric oxide in the brain. Prog. Neurobiol., 76, 126-152, 2005.

18. Hakky T.S., Jain L.: Current use of phosphodiesterase inhibitors in urology, Turk. J. Urol., 41, 88-92, 2015.

19. Hambrecht R. et al.: Correction of endothelial dysfunction in chronic heart failure: additional effects of exercise training and oral L-arginine supplementation. J. Am. Coll. Cardiol., 35, 706-713, 2000

20. Herchuelz A. et al.: Clinical pharmacokinetics of once - daily molsidomine: From immediate - release to prolonged - release once - daily formulations. Am. J. Drug Delivery., 2, 131-141, 2004.

21. Hiremath J.G. et al.: Pharmaceutical aspects of nicorandil. Int. J. Pharm. Pharm. Sci., 2, 24-29, 2010.

22. Horinaka $S$. et al.: Nicorandil protects against lethal ischemic ventricular arrhythmias and up-regulates endothelial nitric oxide synthase expression and sulfonylurea receptor $2 \mathrm{mRNA}$ in conscious rats with acute myocardial infarction. Cardiovasc. Drugs Ther., 18, 13-22, 2004.

23. Hottinger D.G. et al.: Sodium nitroprusside in 2014: A clinical concepts review. J. Anaesthesiol. Clin. Pharmacol., 30, 462-471, 2014

24. Ignarro L.J., Napoli C., Loscalzo J.: Nitric Oxide Donors and Cardiovascular Agents Modulating the Bioactivity of Nitric Oxide. Circ. Res., 90, 21-28, 2002.

25. INOMAX ${ }^{\oplus}$ website [online]. [access: November 13, 2015]. Available at: < http://inomax.com/>.

26. Kannam J., Gersh B.J.: Nitrates in the management of stable angina pectoris [online]. [access: November 13, 2015]. Available at: $<$ http://www.uptodate.com/contents/nitrates-in-the-management -of-stable-angina-pectoris $>$.

27. Kinoshita M., Sakai K.: Pharmacology and therapeutic effects of nicorandil. Cardiovasc. Drugs Ther., 4, 1075-1088, 1990.

28. Kirkebøen K.A., Strand O.A.: Nitric oxide - an important mediator in sepsis? Tidsskr. Nor. Laegeforen., 119, 4061-4065, 1999.
29. Kopkan L., Cervenka L.: Renal interactions of renin-angiotensin system, nitric oxide and superoxide anion: implications in the pathophysiology of salt-sensitivity and hypertension. Physiol. Res., 58, S55-S67, 2009.

30. Kuhl S.J, Rosen H.: Nitric oxide and septic shock. From bench to bedside. West. J. Med., 168, 176-181, 1998.

31. Lakshminrusimha S.: The Pulmonary Circulation in Neonatal Respiratory Failure. Clin. Perinatol,. 39, 655-683, 2012.

32. Lerman A. et al.: Long-term L-arginine supplementation improves small-vessel coronary endothelial function in humans. Circulation, 97, 2123-2128, 1998.

33. Ma F.X., Han Z.C.: Statins, nitric oxide and neovascularization, Cardiovasc. Drug Rev., 23, 281-292, 2005.

34. Maffei A., Lembo G.: Nitric oxide mechanisms of nebivolol. Ther. Adv. Cardiovasc. Dis., 3, 317-327, 2009.

35. Moncada S., Higgs E.A: The discovery of nitric oxide and its role in vascular biology. Br. J. Pharmacol., 147, S193-S201, 2006.

36. Münzel T., Gori T.: Nebivolol: the somewhat-different betaadrenergic receptor blocker. J. Am. Coll. Cardiol., 54, 1491-1499, 2009

37. Münzel T.: Recent findings on nitrates: their action, bioactivation and development of tolerance. Dtsch. Med. Wochenschr., 133, $2277-$ 2282, 2008

38. Nichols S.P.et al.: Local delivery of nitric oxide: targeted delivery of therapeutics to bone and connective tissues. Adv. Drug Deliv. Rev. 64, 1177-1188, 2012.

39. Nossaman V.E. et al.: Nitrates and Nitrites in the Treatment of Ischemic Cardiac Disease. Cardiol. Rev., 18, 190-197, 2011.

40. Orzelska J., Talarek S.: The current approach to the central effects of sildenafil - role of nitric oxide: cGMP pathway. Ann. UMCS Lublin, Sectio DDD, 21, 139-147, 2008.

41. Pacurari M. et al.: The renin-angiotensin-aldosterone system in vascular inflammation and remodeling. Int. J. Inflam, Article ID 689360, doi: 10.1155/2014/689360, 2014.

42. Parker J.D., Gori T.: Tolerance to the organic nitrates: new ideas, new mechanisms, continued mystery. Circulation, 104, 2263-2265, 2001.

43. Reden J.: Molsidomine. Blood Vessels, 27, 282-294, 1990.

44. Rossaint R. et al.: Inhaled Nitric Oxide for the Adult Respiratory Distress Syndrome. N. Engl. J. Med., 328, 399-405, 1993.

45. Schirmer S.H. et al.: Nitric oxide-donating statins: a new concept to boost the lipid-independent effects. Cardiovasc. Res., 94, 395-397, 2012.

46. Scholtholt J. (1985). Molsidomine. In: Clinical Pharmacology of Anti-anginal Drugs. Abshagen U. (editors). Springer - Verlag Berlin Heidelberg; p. 365-377.

47. Stolbach A., Chanmugam A.: Antihypertensive agents, 2015 [online]. [access: November 13, 2015]. Available at: <http://r.wildcatem. com/wp-content/uploads/2014/11/190_Antihypertensive-Agents_ Tintinalli.pdf $>$

48. The Official Website of Nobel Prize [online]. [access: November 13, 2015]. Available at: http://www.nobelprize.org/nobel_prizes/ medicine/laureates/1998/illpres/

49. Wimalawansa J.: Nitric oxide: new evidence for novel therapeutic indications. Expert. Opin. Pharmacother., 9, 1935-1954, 2008.

50. Yaseen H., Darwich M., Hamdy H.: Is Sildenafil an Effective Therapy in the Management of Persistent Pulmonary Hypertension? J. Clin. Neonatol., 1, 171-175, 2012.

51. Zhou L., Zhu D.Y.: Neuronal Nitric oxide synthase: structure, subcellural localization, regulation and clinical implications. Nitric Oxide, 20, 223-230, 2009. 